\title{
Pediatric endoscopic surgery during the ongoing SARS-CoV-2 pandemic
}

\author{
Amulya K. Saxena ${ }^{1}$
}

Published online: 16 March 2021

(c) Crown, corrected publication 2021

The severe acute respiratory syndrome coronavirus 2 (SARS-CoV-2) responsible for the ongoing coronavirus disease 2019 (COVID-19) pandemic poses a critical concern of reinfections that might enable it to become endemic as the disease continues to unleash its wrath in 2021. As compared to the initial global outbreak in early 2020, when there was scarce data on surgical team responses especially with regard to endoscopic procedures; a few months into the pandemic, major endoscopic surgical societies formulated guidelines that were adapted according to the local conditions and guided the reinstatement of endoscopic surgery, albeit restricted to emergency and urgent procedures. In the United Kingdom, the National Health Service (NHS), similar to many other health care systems, has stratified patients by introducing cohort status. Selected patients that could be identified as low-risk cohorts have enabled the resumption of endoscopic surgery for emergency and urgent cases. Elective cases are still not being performed in many countries that are still under national lockdowns as there has been a large-scale reallocation of health care personnel away from operating theaters towards the management of COVID-19 patients.

During the pandemic, high-risk cohorts have also been offered emergency endoscopic surgery in whom clear advantages were seen using this approach, however, with safety guidelines strictly followed by all involved health personnel. The surgical teams have adhered to revised preoperative assessment protocols, updated anesthesia room preparation practices, safe endoscopic surgery guidelines and recovery room precautions. The literature is extensive on endoscopic surgery during COVID-19, but two specific topics, namely insufflated gas and surgical smoke plume have been the

Amulya K. Saxena

amulya.saxena@nhs.net

1 Department of Pediatric Surgery, Chelsea Children's Hospital, Chelsea and Westminster Hospital NHS Fdn Trust, Imperial College London, 369 Fulham Road, London SW10 9NH, UK subjects of focus during the pandemic. With regards to the insufflated gases used in endoscopic surgery, recommendations have included the use of appropriate ports, secure placement of ports to minimize or prevent leaks, minimizing instrument exchanges, maintaining insufflation pressures to a minimum and safe evacuation of pneumoperitoneum via a filtration system before closure, port removal, specimen extraction, or conversion to open. On the other hand, with regards to surgical smoke plume, no study to date has identified SARS-CoV-2 in surgical smoke, and even if detected, has not established whether these viral particles would be infectious. Similarly, there is conflicting guidance in electrosurgery which also has not shown any evidence for an increase in disease transmission. Therefore, due to the lack of data supporting the claim that SARS-CoV-2 spreads through the endoscopic smoke plume, the use of open procedures cannot be justified in patients where endoscopic procedures have offered clear advantages of reduced hospital stay and reduced complication rates. However, on the other hand, also with the paucity of data on this issue, safe smoke plume evacuation using smoke filters or smoke evacuators for procedures generating surgical smoke during endoscopic procedures is warranted.

In the quest to provide a safer environment for the operating room staff during pediatric endoscopic surgery, the role of personal protective equipment (PPE) needs attention. There is no doubt that endoscopic surgery should be performed in full PPE in intermediate- and high-risk cohort groups. However, this has also added an additional level of difficulty during procedures, in terms of surgical team member discomfort when using special protective face masks, and the visual impediment and head clash when using visors especially during neonatal endoscopic procedures where team members are positioned closer to each other around the operating table. Besides facial discomfort with pressure marks on face, nose and ear, as well as the increase in breathing discomfort with the longer duration of use; special protective face masks have also been liable for unclear 
conversations and lack of clarity in understanding verbal exchanges within the operating team. These distracting factors with varying degree of discomfort experienced by the entire surgical team in full PPE have added an extra level of difficulty in longer advanced endoscopic surgical procedures with teams either doffing and redonning after taking a short break to refresh and avoid lapse of concentration or occasionally persisting with the surgery while enduring increasing discomfort. On the other hand, it could be debated upon whether full PPE should not be donned for low-risk neonatal and pediatric endoscopic procedures to overcome the abovementioned disadvantages.

Two notable developments in the past year that have further facilitated a safer environment for operating room personnel working during the pandemic are the improvements and availability of SARS-CoV-2 rapid detection tests since mid-2020, and the successful launch of SARS-CoV-2 vaccines, along with vaccination of health care personnel since December 2020. However, the unavailability and uneven distribution of both the rapid detection test and the vaccine will be major factors responsible for the staggered and uneven return to normalization of endoscopic surgical practice across the globe. Furthermore, the emergence of new SARS$\mathrm{CoV}-2$ variants in different parts of the world and their spread had added a new dimension to the pandemic as the vaccine responses to counteract them still remain unclear.

Education and training in pediatric endoscopic surgery is an area that has been affected during the ongoing pandemic. Due to the previously highlighted precautions and risks of performing endoscopic surgical procedures, in many centers, it is obligatory for procedures to be performed by the
Consultant surgeon to minimize the operating time. This has reduced the hands-on time for surgeons-in-training in pediatric endoscopic surgery for over a year and has stalled their development in many routine procedures. Prior to the pandemic, there were numerous hands-on courses offered to pediatric surgeons-in-training in state-of-the-art skill labs; however, since the onset of the pandemic, these courses could not be organized which has been another setback in hand-on training. Some pediatric endoscopic surgery courses were moved to the virtual platform as Webinars that offered an interactive teaching platform; however, these have been unable to bridge the hands-on skills gap. Many centers were quick to recognize these limitations and set up skill-labs within their institutions and created specific handson models for training on procedures pertinent to pediatric surgical pathologies. Virtual reality simulators have also to a certain extent played an effective role in bridging the loss of training time.

The encouraging circumstances in pediatric endoscopic surgery are the facts that SARS-CoV-2 affects the pediatric age group in lower numbers and with lesser severity when compared to adults, although children of all ages are susceptible. The lower pediatric affection has offered a larger low-risk cohort for emergency and urgent endoscopic surgical procedures. This is especially important in neonates and infants with certain congenital anomalies and pathologies in whom the benefits of endoscopic surgical procedures have been shown to outweigh conventional open approaches.

Amulya K. Saxena

Editor-in-Chief 